\title{
Zhejiang Province Communication Resource Management System 2nd Stage Technology Solutions
}

\author{
Y.Y. Ji, J.J. Yu \\ Zhejiang Communications Administration \\ Hangzhou, Zhejiang, China
}

\begin{abstract}
Zhejiang province communication resource management system project preliminary established the telecommunications infrastructure database, realize the Digitization Management Communication Management Bureau of Zhejiang Province on the communication resources in the province, the tower, the base station, chamber, pipe, bar the way five kinds of infrastructure building and sharing of the electronic office. This year with the broadband China strategy, we fully consider the demand management of communication resources for a period of time in the future, the resource management system is also put forward higher requirements, therefore, based on the project of the business and technical architecture of the system on the two stage of capacity expansion scheme, the functional modules of the system and system network structure is improved.
\end{abstract}

Keywords-communication resource management system; expansion; improvement

\section{INTRODUCTION}

The 1st stage of Zhejiang province communication resource management system had been in smooth operation since 29th October 2010, which realized the electrization of construction and sharing of infrastructure, including tower, base stations, chamber, cable and bar [1]. Consequently,

Communication Management Bureau gained more efficient information methods in regulation because of the standardization of operators' construction and sharing [2].

In 2013, the State Council issued the announcement of issuing 'Broadband China' strategy and implementation program, which required to enhance the broadband access in our country. Considering the improving demands of communication resource management with the future developing business, and in order to efficiently manage base communication resource in Zhejiang, supervise and coordinate the co-construction and sharing between each operator, as well as to informationize the management to operators, it is essential to reform and expand the 1st stage system [3].

\section{THE CURRENT STATUS OF 1ST STAGE SYSTEM AND ANALYSIS}

\section{A. The Current Status of System Function}

The 1st stage system employs multi-layer structure, including Business Showing layer, Application Service layer and Data Service layer. The Business Showing layer is used to achieve business function, Application Service layer is used for basic system services, such as security management, authority management, process management, etc. Data Service layer offers basic system data services [4].

\author{
Y. Lin \\ Zhejiang province public information industry co. LTD \\ Hangzhou, Zhejiang, China
} below,

Currently the system structure is as the figure 1 shown

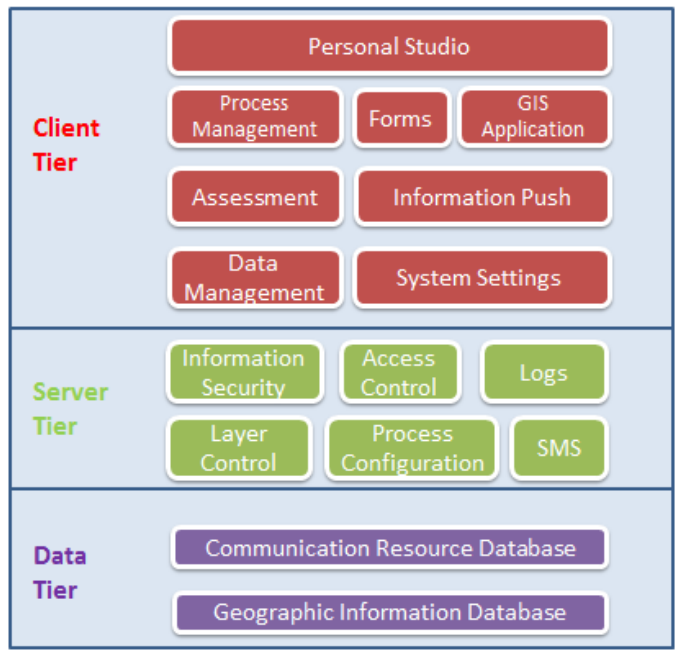

FIGURE I. SYSTEM STRUCTURE AT PRESENT.

B. The Current Status of System Network

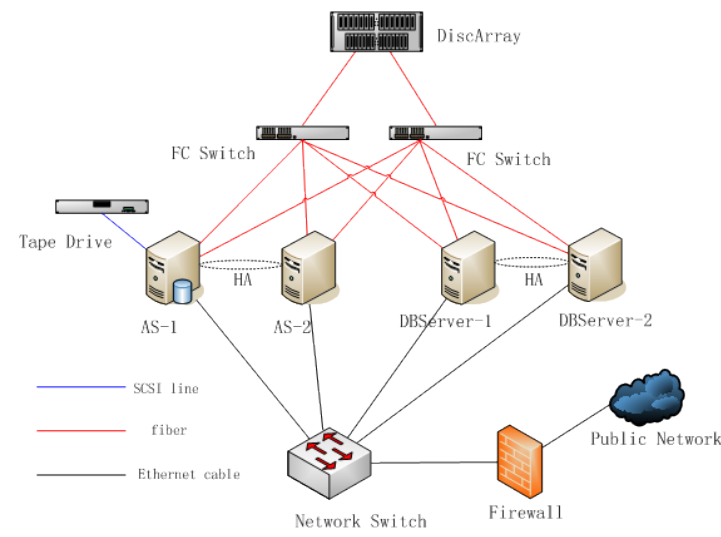

FIGURE II. NETWORK TOPOLOGY AT PRESENT.

As shown in figure 2 above, currently the hardware is consist of 4 servers, in blcluding 2 application server and 2 database server. Those 4 servers access RAID through a SAN switcher, and public network via a firewall.

\section{Analysis of Existing Problems}

Right now there are 4 problems in the system as below, 
1. Expanding of management boundary. So far the bureau is able to supervise towers, base stations, chamber, cable and bar, however residential community is lack of supervision and coordination. Furthermore, it is difficult for the supervisor to forbid unfair-competition in the construction of resident network via information methods.

2. Insufficient system security. Previous system structure can only satisfy demands of these 5 infrastructures. After several module added to the system, it now demands better hardware and network environment. Moreover, in order to improve the security level of system core data, it is estimated that 2nd stage system would store and deploy data individually.

3. Instable VPN. When large amount of user logined via VPN, although it did not beyond the limit of firewall parameter, some branch operator still disconnect on VPN.

4. Insufficient system function. It is impossible to realize the sharing construction in previous system. Besides, enterprises cannot view whether they have accomplished the examination indexes of Ministry of Industry and Information Technology in that. It is necessary to adjust the system with the times.

\section{THE GOAL AND MAIN CONTENT OF 2ND STAGE SYSTEM}

The 2nd stage system will be built and updated at the foundation of existing system, which aims to reach the following goals,

1. Support the supervision of residential network. The system should offer the function of collaborative office on construction of residential network between each enterprise, through an added residential network management module. Moreover, the local communication development office should be able to inquire data of local co-construction and sharing and supervise the process. Finally, this system should offer a whole picture of local construction for supervisor and provincial operators, thus providing statistics and data analysis for relevant management decision and business analysis.

2. Monthly report about co-construction and sharing of 5 infrastructures mentioned above. To extract report more conveniently and quickly, a monthly report about coconstruction and sharing will be developed based on existing infrastructure database. In addition, the previous report about co-construction and sharing of 5 infrastructures shall be reformed to satisfy the requirements of the Ministry.

3. Offer evaluation methods. Construction assessment and rent settlement shall be added to provide valuable data analysis for enterprises and managers. Thereby, this will provide evidence for evaluation of economy and society benefits.

4. Manage construction and commission maintenance of a third party. Provide management for supervisors, on third party screening of construction and commission maintenance. Thus enterprises can report the 3rd party of each projects, all third party will be under unified management of managers.

5. Be adapted to key construction area. To satisfy increasing demands of relevant information, base information management of key area( including 10 key places and 3 construction area) shall be added, and provide summary report in key area.

6. Other function improving. Make optimization and improvements, such as adding early warning system, management of sharing property, national documents and standard library.

\section{THE IMPROVEMENT PROGRAM OF 2ND STAGE SYSTEM}

\section{A. Improvement PROGRAM of System Function Module}

Many function modules shall be added in this project, such as residential network, 3rd party construction and commission maintenance, rent settlement, etc. Thus the integration of function modules and existing system shall be fully considered. The new system structure is shown as figure 3 below,

Black, previous system function

Red, new function added in 2nd stage system

Green, adjusted function in 2nd stage system

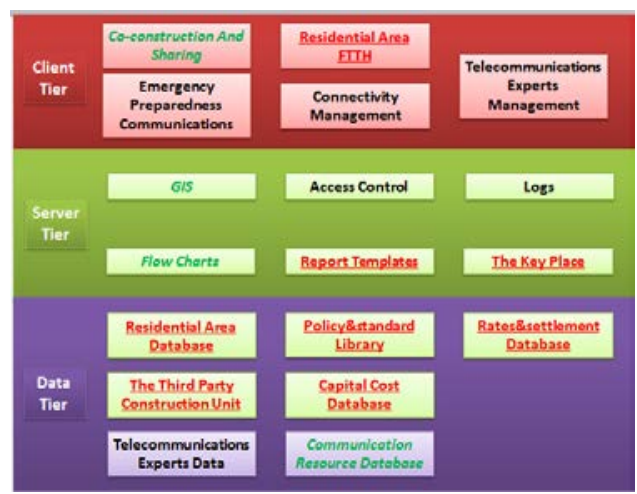

FIGURE III. SYSTEM SOFTWARE STRUCTURE.

Software structure is designed on the base of system security, extendibility, reusability, maintainability and portability. It aims to separate business from system base structure, so that coupling between software modules can be reduced and polymerization will be increased. This new system focuses on business data and is driven by business process, so that it is divided into 2 level, application service layer and business showing layer. Different layers are linked with software interface, which offers running environment for business realization.

In order to satisfy new demands of co-construction and sharing, previous function shall be adjusted to some degree. Besides, this goal shall be reached at least cost, so that new function modules need to be combined with previous function modules to some extent. This article designs the relations between main function modules of application layer and business layer.

\section{B. Improvement Program of System Network Structure}

In order to deal with large amount of subsequent information from each operators, local communication development office and other 3rd party companies and enhance system security, we suggest adding 2 individual servers( HP, double-machine hot standby), 1 RAID. Besides, platform servers are set as backup to each other, so that the system can still work with hot standby technology realized by dual- 
computer software when one node meets failure. The same as network equipment to avoid single points of failure. The improved system network is as figure 4 shown below,

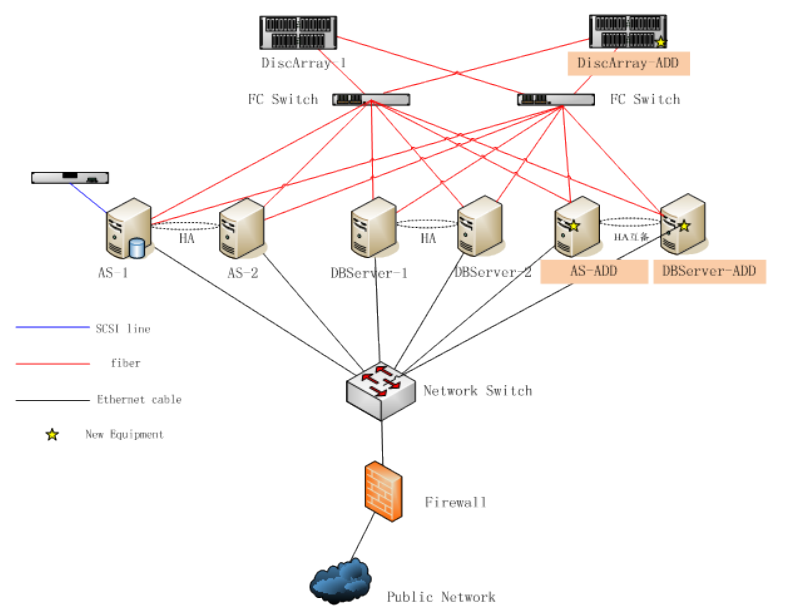

FIGURE IV. IMPROVED SYSTEM NETWORK TOPOLOGY.

In this project, PC servers access the public network via network switcher and firewall (VPN equipment). System users must login VPN to be authenticated by firewall and login the system. Users in public network cannot bypass the firewall and login system intranet without a VPN account and password.

\section{Upgrading Program of System Security}

1. The evaluation of system security. As the servers of database are placed in the public network, so the data safety is very significant. The system has to be evaluated in all aspects, including code safety, database safety, hardware safety, etc. in order to upgrade the system security [5].

2. Targeted repair. System needs to be repaired in a tailored approach after the evaluation. Some common problems in information system are listed below.

3. General security threats. Including many possible situation, such as hackers attacking the network, operating system or application system with malicious code or Trojans; insiders introducing viruses access external network, download/copy software or documents, open suspicious email without authorization; hackers attacking system via a backdoor; accidental operation from authorized users may result in overwritten system documents, data loss or fail.

4. Business information security threats. Including insiders modifying important system data or program via technique or management vulnerabilities without authorization; hackers stealing ID authentication data with all sorts of tools, with those data they can visit network, system or illegally using application software, documents and data without authorization; and hackers visiting network through bypassing security policy and defects in network structure design.

5. Business service guarantees threats. Hackers may cause denial of service through maliciously expending resources of network, operating system and application system with denial-of-service attack tools.

6. Security management threats. Including security risks caused by lack of safe management methods. This can be prevent by patrol supervise and safety strategy, such as periodically changing server password, turning on and off the white list of firewall in time, users regularly adjusting their system password, using China Telecom's UIM to solve network slowness caused by bad connectivity.

7. Hard/software security threats. Including servers' performance overload, hardware failure, accidental system crashes, etc. in order to deal with security threats at this aspect, those problems should be taken into consideration during hardware design period. So dual-computer backup is necessary to keep the system functioning when one server fails.

\section{V.CONCLUSION}

This project is developed on the base of relevant documents issued by Ministry of Industry and Information Technology and demands for Zhejiang communication management Bureau. It will satisfy demands for management in co-construction and sharing under more complicated environment. After the completion of this system, the ability and efficiency of Zhejiang communication management Bureau are improved. Such as co-construction and sharing in telecom infrastructure resource, supporting residential network construction, reducing telecom repeated construction, increasing utilization of telecom infrastructure. Meanwhile this system integrates relevant management process in resource co-construction and sharing, which realizes a coordination work platform and regulates the workflow in resource co-construction and sharing.

\section{REFERENCES}

[1] Qing-zhu Zeng, Research on Telecommunication Infrastructure CoConstruction and Sharing. China Lnternet, 12, 2013.

[2] Jie-juan Zhou, Chang Li, Jie Bu, Application of Co-Constructing and Sharing in the Construction of Customer Premises Network. China Internet, 8, 2013.

[3] Yong-chun Ao, Ming-zhe Hu, The Research on the Mode of Coconstruction and Sharing of the Telecom Infrastructure Based on Operation of the Third-party Companies. Science and Technology Management Research, 32(16), 2012.

[4] Liang Zhang, China's Telecom Database System Optimization Technology Research. Computer Software and Applications, 22, 2011.

[5] Yan Jiao, With Regard to the Status of the Database System Security Research. Network and Computer Security, 5, 2010. 